\title{
Cross-Platform Validation of Notional Baseline Architecture Models of Naval Electric Ship Power Systems
}

\author{
Hasan Ali and Roger Dougal \\ Department of Electrical Engineering \\ University of South Carolina \\ Columbia, SC \\ Email: hasan@,cec.sc.edu
}

\author{
Abdelhamid Ouroua and Robert \\ Hebner \\ Center for Electromechanics \\ University of Texas at Austin \\ Austin, TX \\ Email: r.hebner@mail.utexas.edu
}

\author{
Mischa Steurer, Mike Andrus, \\ James Langston, Karl Schoder, \\ and Rob Hovsapian \\ Center for Advanced Power Systems \\ Florida State University \\ Tallahassee, FL \\ Email: schoder@caps.fsu.edu
}

\begin{abstract}
To support efforts in assessing the relative merit of alternative power system architectures for future naval combatants, the Electric Ship Research and Development Consortium (ESRDC) has developed notional baseline models for each of the primary candidate architectures currently considered, medium-voltage DC (MVDC), conventional $60 \mathrm{~Hz}$ medium-voltage (MVAC), and high-frequency medium-voltage (HFAC). Initial efforts have focused on the development of a consistent set of component models, of which the system models can be comprised, and the basic definition of the system models. The broader objectives of the consortium, however, go beyond the definition of the baseline models. The focus is on the process by which the models are implemented in software and validated, the process by which the performance of the disparate system models are objectively and quantitatively assessed and compared, and, ultimately, the process by which the relative merits of the architectures may be assessed. This paper focuses specifically on cross-platform component validation.
\end{abstract}

\section{INTRODUCTION}

The roles of modeling and simulation $(M \& S)$ in design of future ships [1] is significantly gaining importance as it has tremendous potential in both guiding early stage designs and detailed analysis of design choices without the need for building hardware prototypes. After selecting baseline systems, choices for a set of baselines models have to be made. The models should then facilitate studies to compare the merits of power system options. The efforts reported here concern the three contenders in building the next generation U.S. Navy ships [2]. These three architectures represent the baseline modeling effort with the goals of being a point of reference and should not be used for direct comparison between architectures. Any real engineering design will be preceded by an optimization process which was not applied in

This work was supported in part by the Office of Naval Research through grant N00014-08-1-0080. this case. The implementation targets multiple software platforms for cross-platform validation.

The fundamental topology used by each of the baseline models is illustrated by Figure 1, which is primarily based upon the information originally provided in the Roadmap for the Next Generation of Integrated Power System Technology [2]. In this topology, four turbo-generators are connected to a ring-bus that supplies two propulsion power trains, four ship service zonal loads, a radar load, and a pulse load. A bulk energy storage system is also connected to the distribution bus for Uninterruptible Power Supply (UPS) and/or ride-through power capabilities. The system models have been created with component models that reflect the common features of load demand and generation portfolio while taking advantage of the architectures' benefits.

Each of the component models, and subsequently, each of the system models are initially implemented in two different tools, MATLAB/Simulink with SimPowerSystems and PSCAD. For each of the models, the intended process is to identify operational scenarios to provide insight into model behavior, and identify potentially influential model parameters and response variables for each of the scenarios. The models are evaluated over a region within the parameter space, and quantitative metrics used in assessing the behavior of the models and the agreement between the implementations thereof. These steps are important for understanding the behavior and parametric sensitivities of the models over wide ranges of parameter values, as well as for cross-validation of the models. Further, this process will help to establish procedures that allow for comparison and exchange of system models among different entities. 


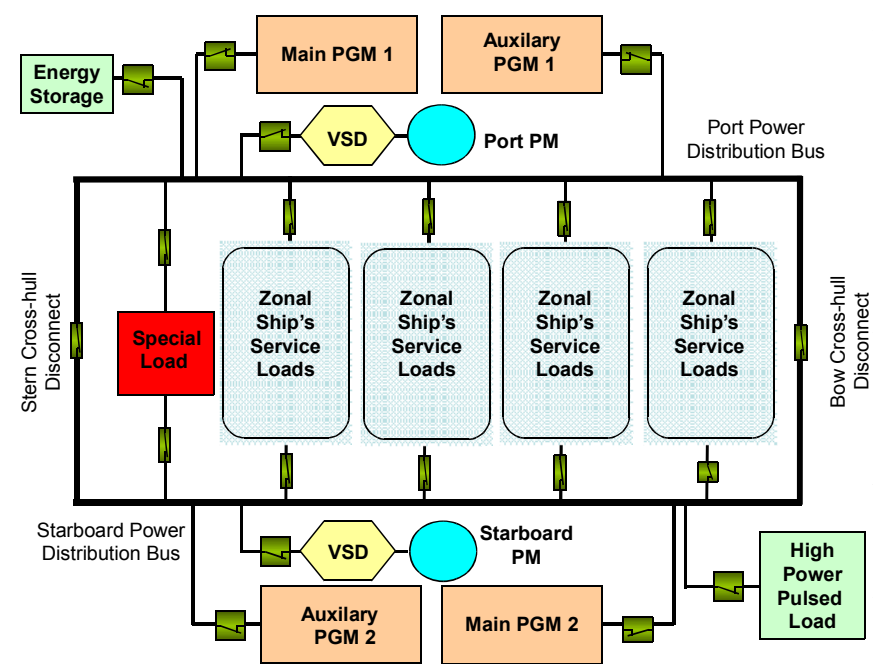

Figure 1. General topology for notional baseline system models (PGM-Power Generation Module, VSD — Variable Speed Drive, PM—Propulsion Motor)

The objectives in this effort go beyond the definition of the baseline models. The focus is on the process by which the models are implemented in software and validated and the process by which the performance of the disparate system models are objectively and quantitatively assessed and compared. Establishing rigorous approaches for these aspects is paramount to enabling an increased role of $M \& S$ in the design process and, thus, enabling the envisioned benefit of reduced development costs and time for future Navy ships.

This work focuses primarily on the process by which instances of component and system models implemented for different software platforms are used for cross-validation against each other. As one of the key objectives of the process is to ensure consistent predictions from models implemented in different software platforms, the methods for crossvalidation between model implementations form an essential part of the framework. The approaches to cross-validation of the simulation models and experiences with the application of the approaches in implementation of the baseline systems are addressed in the following sections.

\section{PROCESS FOR VERIFICATION AND VALIDATION}

A number of guidelines and recommendations related to verification and validation have been proposed by various organizations and research communities such as the Society for Computer Simulation, the IEEE, the United States Department of Defense (DoD), the American Institute of Aeronautics and Astronautics (AIAA), and the American Society of Mechanical Engineers (ASME), a general summary of which is provided by [3]. However, [3] points out that, while there are important points to draw from each of these sources, there are different motivations and intended applications among them and a distinction and limitation of scope is made for verification and validation of scientific models. For example, while the IEEE definitions of verification and validation, which tend toward general software development activities, focus on adherence to defined requirements, validation of scientific models is concerned with assessing the degree to which a simulation model properly represents the physical system upon which it is based. Similarly, while many simulations in such fields as operations research, for example, may involve modeling of complicated sentient entities such as governments, societies, or economies, scientific computing models are generally limited in scope to physical systems. It is with this more limited scope and intent of validation of scientific models that छefforts discussed herein are aligned. In this context of Everification and validation of scientific models, the primary 产objectives are quantification of sources of error in the simulation, such as discretization and truncation error, quantification and propagation of uncertainty, and comparison with experimental results in the context of the known error and uncertainty. As it is noted in [3] that scientific models generally cannot be universally validated, the process of validation is an ongoing one, aimed at presenting a body of evidence attesting to the accuracy of the model in the context of which it has been evaluated.

A major motivation of the development of the baseline models was to explore the application of available model validation methods to electromagnetic transient simulations of shipboard power systems, one of the common types of simulations employed in ESRDC activities [4]. Although development of the baseline models has progressed, application of the verification and validation methodologies to the component models is only in the early stages. Much work remains in assessing the suitability of many of the methods, as well as identifying additional techniques which may be needed. One of the challenges is the need to model large, expensive, unrealized systems, for which experimental data for validation is scarce or nonexistent. Further, the modeling efforts, particularly at the level of the shipbuilders, will almost certainly involve the use of vendor-provided "black box" component models, for which the modeling equations and source code will not be readily available to the system modeler. This may present difficulties for quantification of simulation error, as these techniques typically require some assessment of the exact solution to the governing equations. In this respect, validation activities at the component level may be of particular importance, and may require validation over a wide range of the parameter space for the models in order to account for the range of surroundings in which the models may be used. Initial work in verification of the component models is presented, primarily focusing on cross-platform verification. This is an important step toward establishing a suitable process for validation of models developed in the course of the work conducted.

\section{VERIFICATION OF COMPONENT MODELS}

\section{A. Propeller and Hydrodynamics}

The general model employed for the twin propeller system and hydrodynamics is based on the model detailed in [5], and is illustrated by Figure 2. Each of the propellers is coupled to a propulsion motor model, from which the shaft speed, $\omega_{\text {prop }}$, is received as an input. Each of the propeller models also receives feedback of the ship speed, $V_{\text {ship }}$, from the ship hydrodynamics model, and computes the thrust, $F_{\text {ship }}$, on the ship and counter-torque, $T_{p r o p}$, on the motor shaft as functions of the ship speed and propeller speed. The hydrodynamics 
model accounts for the mass of the ship, $m_{\text {ship}}$, and the hydrodynamic resistance, $F_{d r a g}$, in order to determine the ship speed. Although the model is based on [5], notional data sets have been employed for the models such that these are more representative of a destroyer surface combatant.

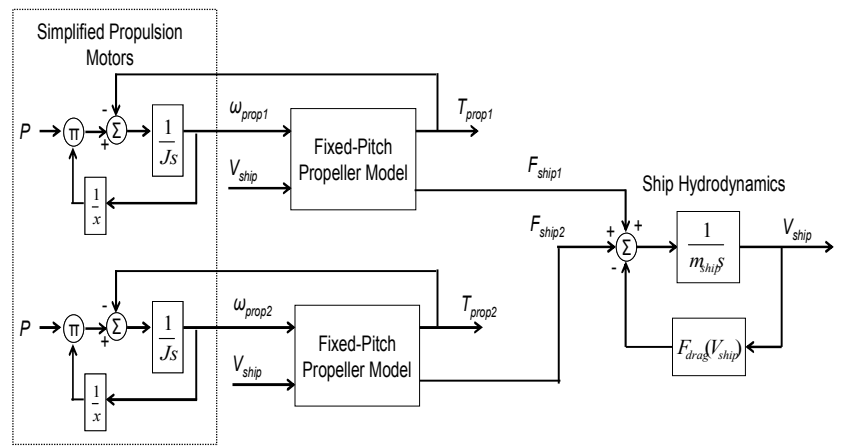

Figure 2. Propeller and hydrodynamics model

For verification testing of this subsystem, simplified propulsion motors with reasonable moments of inertia, $J$, were assumed for the surroundings for the model, as illustrated by Figure 2. In addition to steady-state testing of the model, a dynamic scenario was considered in which the system was brought to steady-state with some initial input propulsion power, and then a step increase in propulsion power was applied. The propeller speed and torque, along with the ship speed, were monitored as the system was allowed to reach a new steady-state point, as illustrated by Figure 3. The timedomain waveforms were summarized by a set of scalar response variables, including the initial and terminal values, rise time, and settling time (here defined to be the time to settle within $2 \%$ of the final value) of each quantity.

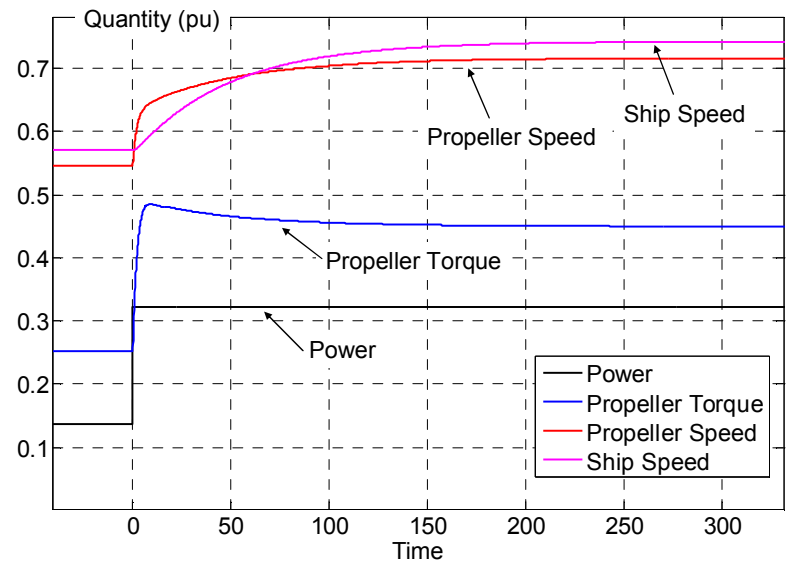

Figure 3. Results from a step increase in propulsion power

Allowing the initial propulsion power to vary between 0.1 and $0.8 \mathrm{pu}$ power, and allowing the step in power to vary between 0.05 and 0.2 pu power, an ensemble of twenty sets of parameter values for the scenario was selected based on a random Latin hypercube sample, in order to provide some general coverage of the parameter space, as illustrated by Figure 4. Both the Simulink and PSCAD cases were evaluated for each set of parameters for the scenario, and the results were compared in terms of the identified response variables in order to verify consistent implementation of the model in both platforms. For each set of parameter values and each response variable, the deviation between the responses from the two models was computed as the magnitude of the difference between the results from the platforms, normalized by the results from the Simulink model.

The results of the comparison for the settling time of the ship speed are illustrated by Figure 5. In Figure 5, the settling time of the ship speed from the Simulink model is plotted on the horizontal axis, with the corresponding result for the respective evaluation of the PSCAD model plotted on the vertical axis. In this case, the model implementations show very close agreement, with the points falling very close to the unity-slope line through the origin. The mean and maximum values of the normalized deviations are also small, reflecting good agreement throughout the parameter space for the scenario. In general, the implementations showed agreement for each of the response variables studied for this scenario to within 1\%. The good agreement between the models is expected, as this model does not make use of any elaborate native models of the software packages. Nevertheless, it is important to verify that the models exhibit consistent behavior to ensure the assumption of agreement is, in fact, correct. However, the results of this exercise do not imply that the propulsion model itself has been validated, since no comparison with actual field data was performed.

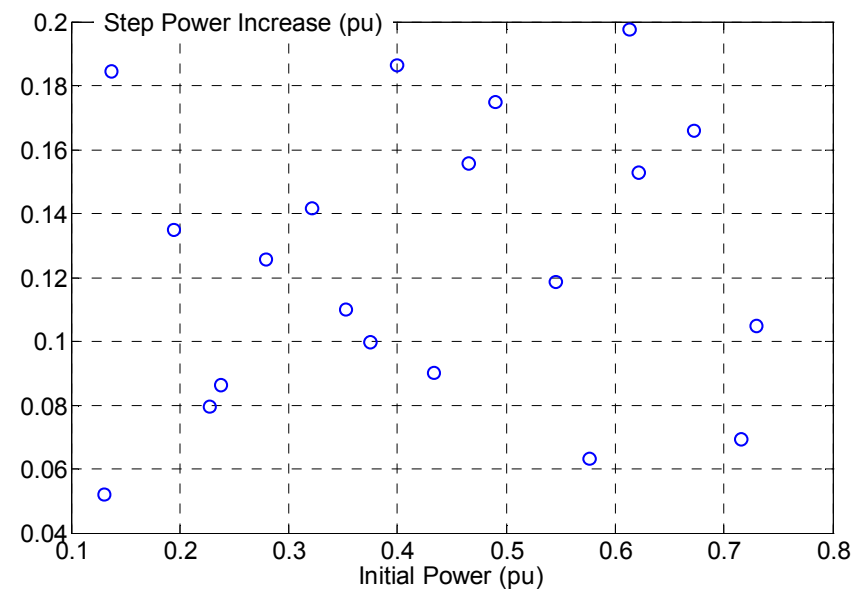

Figure 4. Parameter values at which the simulation models were evaluated 


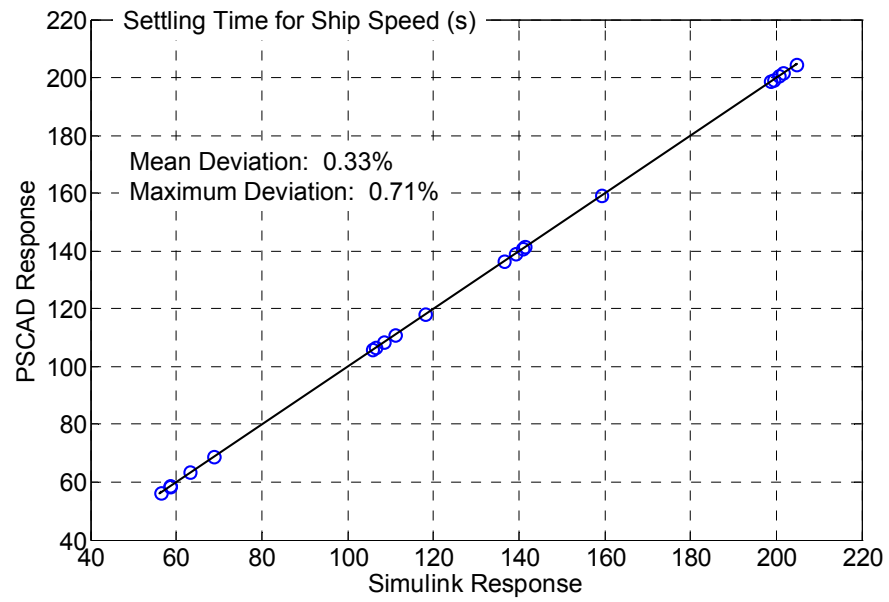

Figure 5. Comparison of results for ship speed settling time

\section{B. Generation Modulet for MVDC System}

The general model for the generation module employed for the MVDC system is composed of a gas turbine for the prime mover, a synchronous machine, a voltage regulator/exciter, and a diode rectifier, as illustrated by Figure 6. In this case, the voltage regulator is used to regulate the $5 \mathrm{kV}$ DC bus voltage, $V_{d c}$, through the synchronous machine excitation voltage, $E_{f}$. One of the key considerations when comparing the consistency of implementation of the model between the platforms is the use of synchronous machine models which are native to the respective platforms, as slight differences in implementations of these components may lead to significant differences in the behavior of the subsystem.

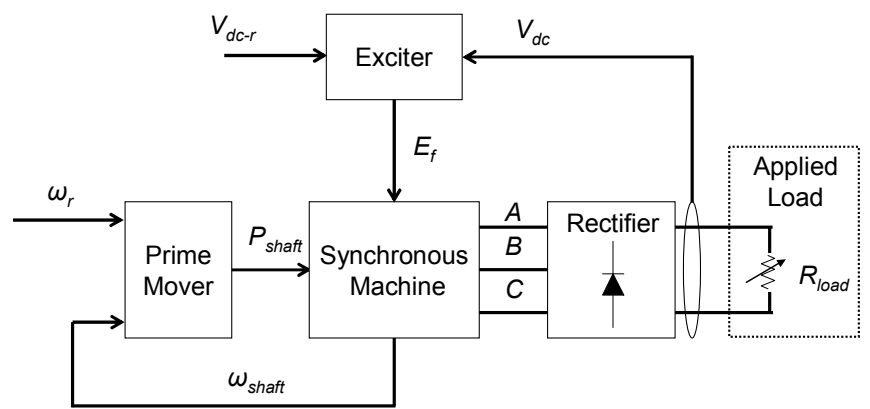

Figure 6. Generator set model for MVDC system

In order to exercise the model, an adjustable resistive load, $R_{\text {load }}$, is placed on the DC terminals of the rectifier, and a scenario is considered in which the system is brought to steady-state at 5\% loading and a step change in load power is applied. In this case, only the magnitude of the load step is varied as a parameter, and a simple sweep of the step power is conducted up to $40 \%$ power. A comparison of the DC voltage and turbine speed for the Simulink and PSCAD implementations is illustrated by Figure 7 for a step of $28 \%$ power. A notable difference in the behavior of the models is evident even at this level of stepped power. However, of particular interest is the much more significant discrepancy in the behavior of the models for a larger step in power of $37 \%$, as illustrated by Figure 8 .
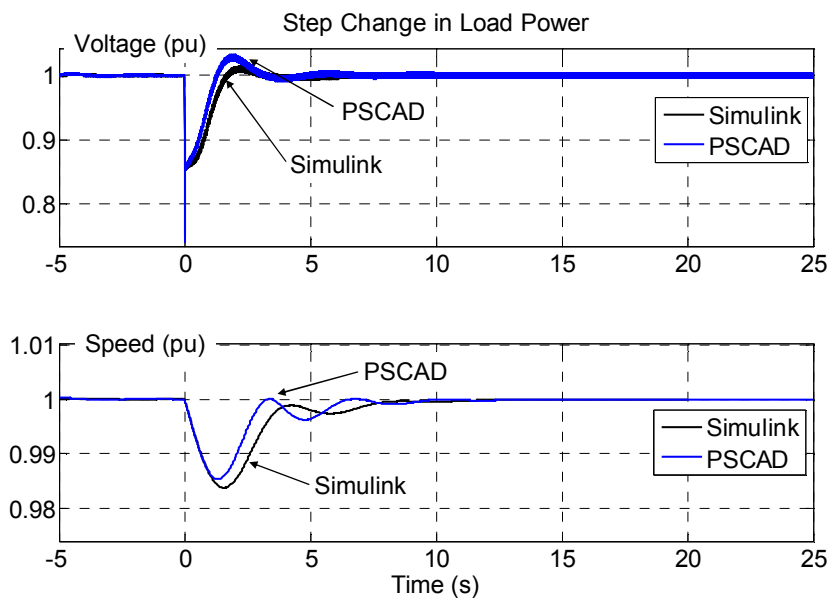

Figure 7. Comparison of results for $28 \%$ step increase in load power
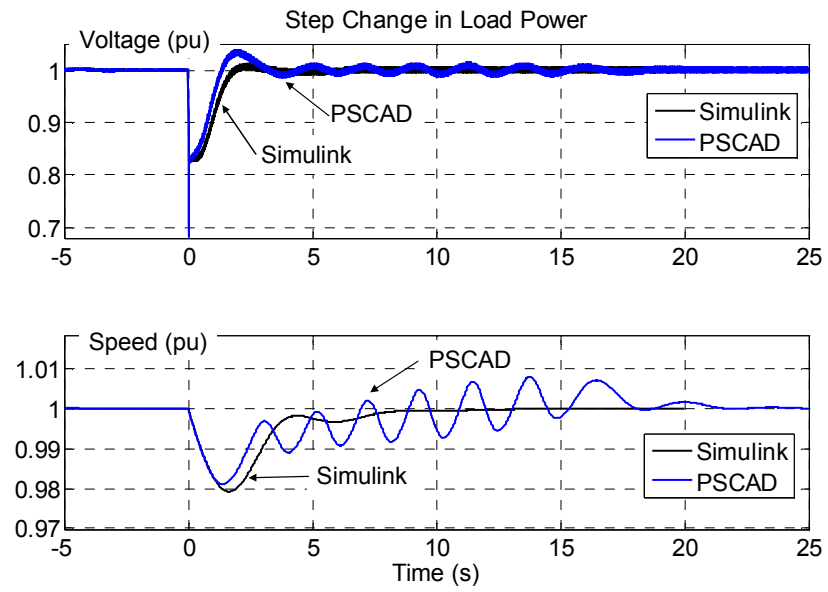

Figure 8. Comparison of results for $37 \%$ step increase in load power

In order to study the behavior of the models, the time-domain waveforms were summarized through a set of response variables including initial and final steady-state real and reactive power, DC voltage, excitation voltage and current, and turbine speed. Other response variables included the maximum deviation from nominal of the DC voltage and turbine speed, and the settling time of the DC voltage, turbine speed, and excitation voltage and current. The response of the models in terms of the settling time for the DC voltage (here defined to be within $1 \%$ of nominal) is illustrated by Figure 9 . Figure 9 shows reasonable agreement for the settling time for most of the model evaluations, but shows a significant discrepancy for the case in which a $37 \%$ step in power was applied. 


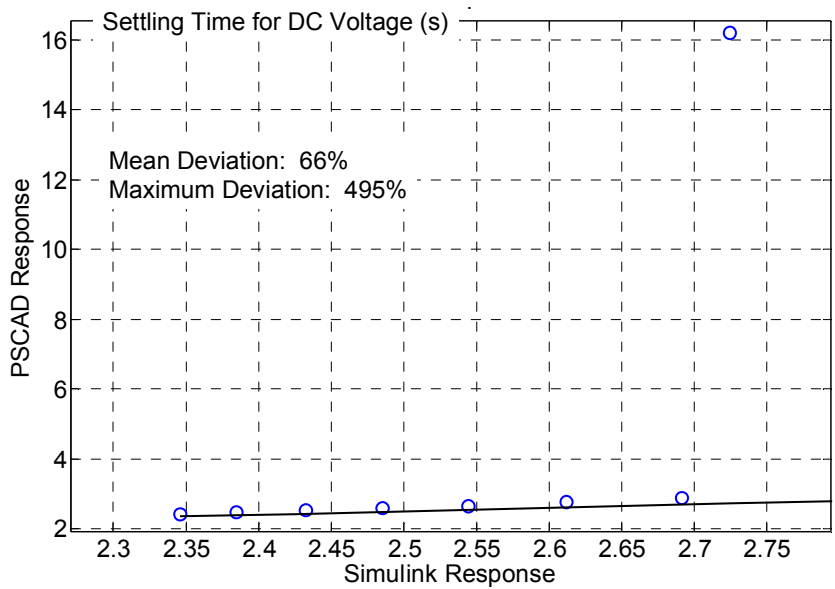

Figure 9. Comparison of results for $37 \%$ step increase in load power

These results are important and guide the model developer to further investigate the discrepancies between the models in order to eliminate this obvious inconsistency in implementation. Hence, this serves as a reasonable example to illustrate the process by which the models are being scrutinized in order to verify consistent implementation. Although this demonstrates a rather trivial case in which only a single parameter was varied at a small number of values, the approach of comparing scalar response variables can be particularly useful for cases in which a large number of model evaluations would preclude inspection of each set of timedomain results.

\section{Supercapacitor Energy Storage in MVAC system}

Figure 10 shows a $4.16 \mathrm{kV} / 60 \mathrm{~Hz}$ ac source, an energy storage system (ESS), and a test load connected to a grid point through breakers eBrk1, eBrk2, and eBrk3, respectively. The energy storage system consists of a Wye-Wye 4.16/0.45 kV transformer, a pulse width modulation (PWM) converter using insulated-gate-bipolar-transistors (IGBT), a DC-DC (buckboost) converter using IGBT, and a supercapacitor. The PWM-converter and the DC-DC converter are connected through a dc link capacitor $\mathrm{C} 1$. Labels eVc, eid, eV0, and ei0 indicate the voltages across the supercapacitor, output current of the supercapacitor, voltage across the dc link, and inverter current, respectively. The ESS inverter gate signal (gDischgInvP) and the DC-DC converter gate signals (gchgP for charge and $\mathrm{gDischgP}$ for discharge) are determined according to control logics.

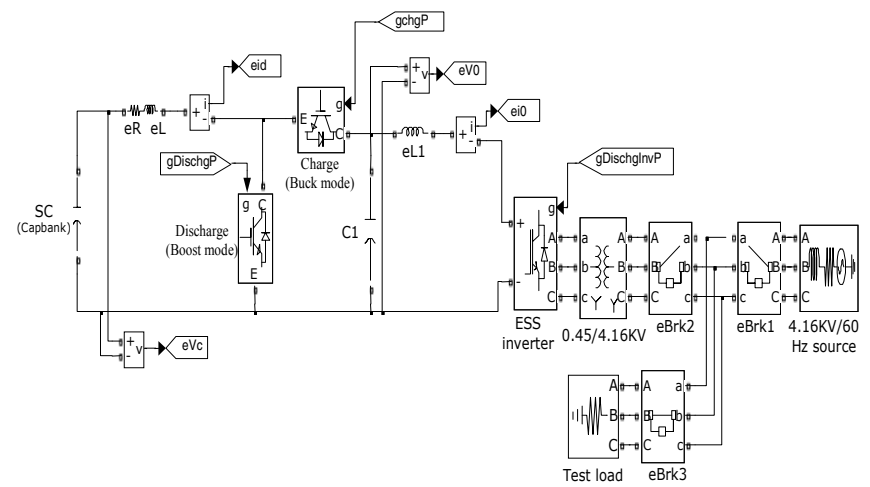

Figure $10.4 .16 \mathrm{kV} / 60 \mathrm{~Hz}$ ac source with an energy storage system (ESS) and a test load
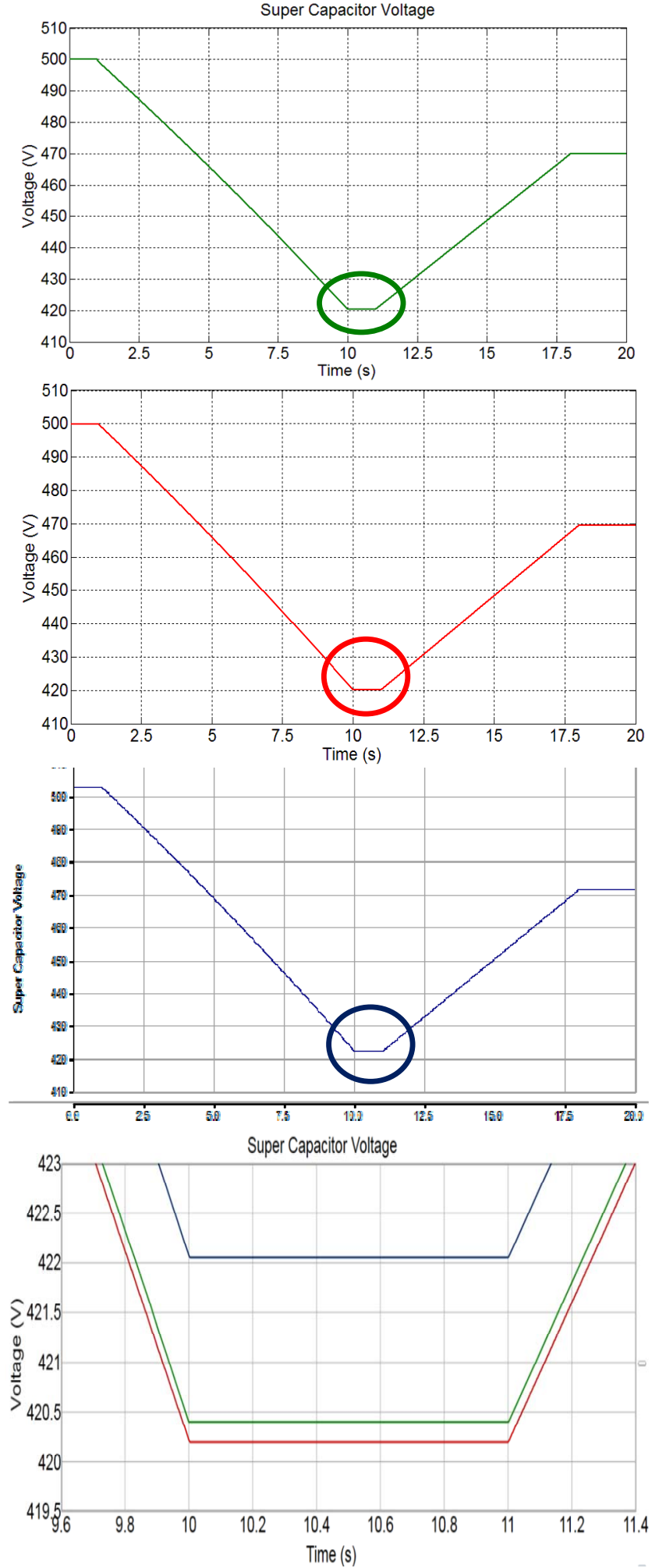

Figure 11. Voltage responses of supercapacitor energy storage: Simulink, VTB, PSCAD, zoom-in/detail (top to bottom)

Figure 11 shows the supercapacitor voltage responses using three simulation platforms, namely, Matlab/Simulink, VTB, and PSCAD. It is shown that the supercapacitor was initially charged at $500 \mathrm{~V}$, and from 1 to 10 seconds it is 
delivering power to the grid. Then from 11 to $18 \mathrm{~s}$, the capacitor is charged by the grid supply. It is noteworthy that the performance of the supercapacitor in all three platforms is almost the same. The $2 \mathrm{~V}$ offset in the PSCAD result is due to a modified charging voltage reference.

\section{DISCUSSION}

The development models electric shipboard power systems in sufficient detail to allow judgment of many system performance issues before building prototypes. The difficulties encountered in the modeling include the need for parametric studies of system models even though each individual system instance is computationally expensive, i.e., requires an execution time on the order of hours. Additional work in formulating an appropriate process will address assessing discretization error, obtaining experimental data for validation, and further comparison of architectures by defining appropriate, universally applicable scenarios and system response quantities. Two important points to address in model validation are:

- $\quad$ Generic package-supplied models of equipment (such as a synchronous generator) may have subtly different mathematical representations and those equations may be solved by different computational algorithms in the different simulation environments. Are these differences small enough that meaningful conclusions can still be drawn from system-level comparisons, regardless of which simulation package is used?

- Generic models must be parameterized in order to represent specific equipment. To what accuracy can properly parameterized generic models actually represent some range of possible physical implementations of that equipment, over a range of sizes (e.g. synchronous generators over power range from $10 \mathrm{~kW}$ to $100 \mathrm{MW}$, or inverter constructed with IGBTs vs IGCTs). Are these generic models sufficient to provide useful (correct) guidance for high-level system architecture decisions, even though they might not accurately represent a particular implementation of a component that will later be put into that system?

Currently, one instance of each system architecture has been designated as the architecture's baseline system. It is understood that alternative configuration options exist and will have to be evaluated before deciding on an optimal power architecture for a new ship considering aspects such as cost, size, weight, performance, efficiency, vulnerability, and survivability. The appropriateness of levels of detail of system representation in simulation needs to be considered before proceeding to use models as specification for selecting an architecture and actual hardware. Finally, rigorous cross-platform validation of the system models has yet to be performed.

\section{CONCLUSION}

The ESRDC is currently undertaking modeling and simulation efforts to establish notional baseline models of three electric shipboard power system architectures, MVAC $(60 \mathrm{~Hz}), \mathrm{MVDC}$, and HFAC $(>60 \mathrm{~Hz})$. These models are available to university researchers, the Navy, and the shipbuilder community alike for benchmark computations, enabling objective comparisons of solution architectures and models implemented on different platforms. This work focuses on the validation processes applied to component (or sub-system) models developed in different simulation platforms. The progress made covers the early stages of component testing and building first versions of complete systems. Next steps will open up the models to a larger group of researchers for increased scrutiny and an attempt in revising component models for consistency.

\section{ACKNOWLEDGMENT}

The authors would like to thank Isaac Leonard, Tim Chiocchio, and Omar Faruque of the Florida State University Center for Advanced Power Systems for their contributions to the modeling effort.

\section{REFERENCES}

[1] Moh. Hasan Ali, Roger A. Dougal, M. Steurer, L. Graber, M. Andrus, D. Infante, J. Ciezki, R. E. Hebner, A. Ouroua, D. Weeks, "'Guidelines for the Specification of Models to be Used in Design-Oriented Simulations," in Proceeedings of the GCMS, Ottowa, Canada, July 11-14, 2010.

[2] N. Doerry, Next Generation Integrated Power System, NGIPS Technology Development Roadmap, Naval Sea Systems Command, 2007.

[3] W. L. Oberkampf and C. J. Roy, Verification and Validation in Scientific Computing, New York, Cambridge University Press, 2010.

[4] Electric Ship Research and Development Consortium (ESRDC), http://esrdc.caps.fsu.edu/

[5] E. J. Lecourt, Jr., "Using Simulation To Determine the Maneuvering Performance of the WAGB-20," Naval Engineer's Journal, January 1998, pp. 171-188. 